\title{
Experience-Consistent Fuzzy Rule-Based Systems: An Enhancement of Data-Oriented Fuzzy Modeling
}

\author{
Witold Pedrycz \\ Department of Electrical \& Computer Engineering \\ University of Alberta, Edmonton AB T6R 2G7 Canada \\ and \\ System Research Institute, Polish Academy of Sciences \\ Warsaw, Poland \\ pedrycz@ee.ualberta.ca
}

\begin{abstract}
Nowadays fuzzy modeling is dominated by data-driven constructs. The resulting granular constructs (say, fuzzy rules) are developed on a basis of numeric data. The genuine challenge arises when the available data become very limited and/or noisy so that it becomes evident that the quality of the constructed model could be quite low. If some domain knowledge has been acquired in the past and becomes now available in the form of some fuzzy models, its prudent usage could be highly advantageous. In this study, we assume that such domain knowledge is captured in the form of some other rule-based topologies constructed on a basis of some previously available data sets (which however cannot be accessed explicitly). To emphasize the very nature of system modeling being guided by this form of the reconciliation mechanism, we refer to the resulting methodology as experience-consistent fuzzy system identification. By coming up with a certain augmentation of the optimized performance index, it is demonstrated that the domain knowledge captured by the individual rule-based models play a similar role as a regularization component typically encountered in system identification. Detailed algorithmic considerations embrace several design scenarios in which we apply the mechanism of experience consistency at the level of conditions and conclusions of the rules. We also show that a level of achieved experience-driven consistency can be quantified through fuzzy sets (fuzzy numbers) of the parameters of the local models standing in the conclusion parts of the rules this leading to the emergence of granular constructs of fuzzy modeling.
\end{abstract}

